\section{MS34-P6 Crystal engineering of the Drotaverin salts}

Tamás Holczbauer ${ }^{1}$, Máté Szabó ${ }^{1}$, Mátyás Czugler ${ }^{1}$

1. Research Group of Chemical Crystallography,Institute of Organic Chemistry, Research Centre of Natural Sciences, Hungarian Academy of Sciences, Magyar Tudósok körútja 2., Budapest, H-1117, Hungary

email: holczbauer.tamas@ttk.mta.hu

Structure determinations of drug molecules are essential for the exploration of their physical and chemical properties. Their crystalline state controls their solubility, absorption and other solid state properties thus their structures may provide a wealth of further information.

Drotaverin salts are exemplary for their good inclusion ability of crystallization solvents, so they could provide some interesting contexts by their solubility and also by the rationalization of their solid state structures. The structure was analysed by X-ray diffraction, providing the basic scrutiny in the solid state.

Starting from the drotaverin hydrochloride salt we made more than a dozen crystalline complexes. Besides the numerous solvent crystals we determined the crystal structures of co-crystals and the crystal structure of the hitherto unknown pure salt even. Anion metathesis reactions were also observed during some crystallization.

These crystal structures may provide basis not only for systematization but also means for a conscientious manipulation of salt - like drug forms in general.

These crystal structures give opportunity to observe the conformational changes and the idiosyncrasy of secondary interaction. Maybe these monitorings forecast the new partners of crystallisation.

Keywords: crystal engineering, secondary interactions, salts

\section{MS34-P7 Structural diversity in pharmaceuticals: various sulfonate salts of agomelatine}

Eliška Skořepová ${ }^{1}$, Michal Hušák ${ }^{1}$, Luděk Ridvan ${ }^{2}$

1. Department of Solid State Chemistry, University of Chemistry and Technology Prague, Technicka 5, Prague 6, Czech Republic

2. Solid State Development, Zentiva k.s., U Kabelovny 130, Prague 10, Czech Republic

email: eliska.skorepova@vscht.cz

The search for new solid forms of an active pharmaceutical ingredient (API) is an important step in a drug development. Often, an API has a low water solubility, which then leads to a low oral bioavailability. The problem can be solved by salt formation. One such API is agomelatine (AG), a melatonergic antidepressant. However, agomelatine is an amidic compound and, since amides are considered very neutral, it was quite a surprise, when agomelatine, in the combination with three different sulfonate acids, produced salts. The five novel resulting crystalline forms were AG hydrogensulfate, AG hydrogensulfate methanol solvate hemihydrate, AG mesylate, AG mesylate monohydrate and AG besylate. Their structures were solved either from single-crystal or powder x-ray diffraction data. Interestingly, the structures of the two hydrogensulfates were determined from the same crystal via single-crystal to single-crystal transformation. In all of the structures, the agomelatine molecule was positively charged. Specifically, the amide oxygen was protonated. The proton transfer and the salt formation were also confirmed by solid state NMR and the $\Delta \mathrm{pK}$, calculation.

This work was supported by the Grant Agency of Czech Republic, Grant no. 106/14/03636S and received financial support from specific university research (MSMT No 20/2015).

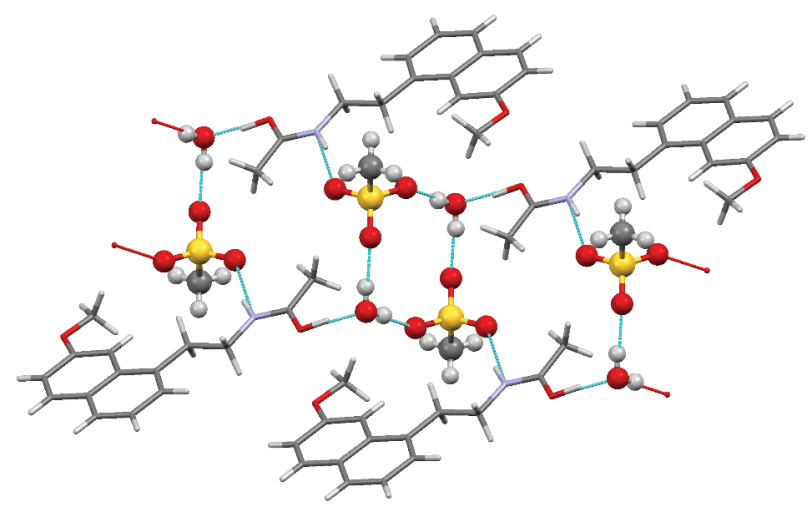

Figure 1. H-bonding in agomelatine mesylate monohydrate

Keywords: x-ray crystallography, pharmaceuticals, salt formation 